\title{
Automated, accurate and fast segmentation of 4D cardiac MR images
}

\author{
Jean Cousty ${ }^{1}$, Laurent Najman ${ }^{1}$, Michel Couprie ${ }^{1}$, \\ Stéphanie Clément-Guinaudeau ${ }^{2}$, Thomas Goissen ${ }^{2}$, Jerôme Garot ${ }^{2}$ \\ ${ }^{1}$ Institut Gaspard-Monge, Laboratoire A2SI, Groupe ESIEE, France \\ $\{j$.cousty, 1.najman, m.couprie\}@esiee.fr, \\ ${ }^{2}$ CHU Henri Mondor, Créteil, France
}

\begin{abstract}
Based on discrete mathematical morphology, we propose a new automated and fast procedure to segment the left ventricular myocardium in $4 \mathrm{D}(3 \mathrm{D}+\mathrm{t})$ cine-MRI sequences. Thanks to the comparison with manual segmentation performed by two cardiologists, we demonstrate the accuracy of the proposed method. The relevance of the ejection fraction and myocardium mass measured from segmentations is also assessed. Furthermore, we show that the proposed 4D procedure allows to keep the temporal coherency between the successive 3D segmentations obtained along the time sequence.
\end{abstract}

\section{Introduction}

In cardiology, the precise information on both the dimensions and function of the left ventricle (LV) is essential, either in clinical applications -diagnosis, prognostic, therapeutic decisions- or in the research fields.

Thanks to 3D images at different times of the heart cycle, Magnetic Resonance (MR) imagery allows of a complete morphological LV characterization. The precision on the measures extracted from MR images has been demonstrated [1] and makes MR imagery a "gold standard" for LV analysis.

However, due to the considerable amount of available data, the analysis and, in particular, the segmentation of such images is fastidious, time consuming and error-prone for human operators. Automated segmentation of cardiac images has shown to be a challenging task.

Since the 90's, many approaches for solving this problem have been proposed, notably in the framework of deformable models [2-4]. From a time series of 3D images of the LV, most of the existing methods iteratively segment each 3D image independently [5-7]. Despite a constant improvement in the accuracy of the produced segmentations, the temporal consistency of the resulting segmentations, a desired feature in the case of a time series of 3D images, is not taken into account by these approaches. Only a few methods consider the time series as a whole 4D image. Although it uses a 4-dimensional atlas, the method in [8] assigns independently each pixel to one of the objects and does not take into account global properties (e.g. connectedness or presence of holes) relative to the 
spatiotemporality of the produced segmentations. In [9], the authors propose a 4D deformable model with temporal constraints. Despite promising results on synthetic SPECT images, the resulting segmentation scheme was not fully assessed on real cardiac images.

In order to take into account the time continuity in $4 \mathrm{D}$ cine-MRI, we investigate a new LV segmentation scheme based on discrete mathematical morphology. This theory [10] consists of analyzing geometrical objects through their interaction with predefined geometrical shapes. The notions of neighborhood and connectivity are the basis of this framework. In particular, operators dedicated to image segmentation are extended to 4-dimensional spaces by defining elementary 4-dimensional neighborhoods.

The main features of the LV segmentation method proposed in this paper are: $(i)$, accuracy assessed against manual segmentations performed by two cardiologists; $(i i)$, resulting segmentations taking into account spatiotemporal properties; (iii), automated and fast; and (iv) few parameters whose setting relies on physical and anatomical facts. In this paper, the general idea of the method is described; more details will be provided in a forthcoming extended version.

\section{Segmentation method}

The segmentation scheme in mathematical morphology [11] comprises three main steps: recognition, delineation and smoothing. Recognition is the process of determining roughly the whereabouts of the object. Delineation consists in the precise spatial localization of the object borders. Smoothing can be defined as the process of matching the smoothness properties of the segmented object with the $a$ priori smoothness properties of the ground truth.

The recognition process consists in finding markers (landmarks) for each object to be segmented. This step of the method allows prior information to be taken into account in the segmentation result. This prior knowledge derives from the modeling of the objects which are to be segmented. The delineation process, which is generally performed by watershed algorithms, looks for divides, localized on the "most contrasted areas" of the image, which separate the selected markers. Finally, the smoothing step filters the objects obtained after the delineation process by removing their non-significant parts, with respect to prior knowledge about the shape of the objects. The left ventricular myocardium $(L V M)$ is delimited by two surfaces: the epicardial border $(E p B)$ and the endocardial border $(E n B)$. We consider (Fig. 1a): $(i)$, the left ventricular chamber (named $L V C$ ) delimited by $E n B$ and surrounded by $L V M$; and (ii), an object called $L V C M$, made by the union of the $L V C$ and $L V M$, delimited by $E p B$ and surrounded by $L V B$, the Left Ventricular Background which is the complementary set of $L V C M$.

The $L V C$ is a connected set whose intensity is very high and which is surrounded by a significantly darker zone (see Fig. 1b). It is known, from anatomical description, that the $E n B$ is an irregular surface.

The left ventricular myocardium surrounds the $L V C$ and its intensity is darker 


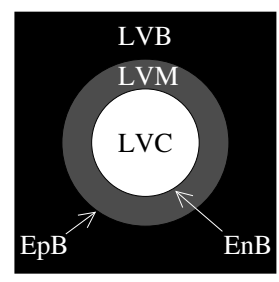

(a)

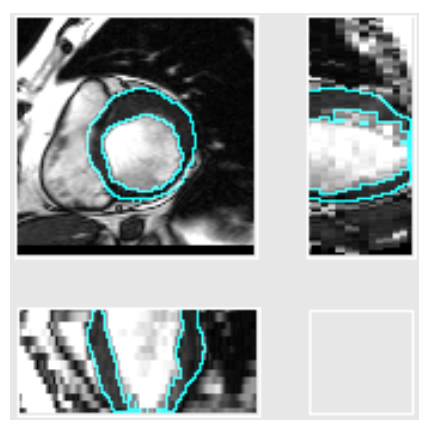

(b)

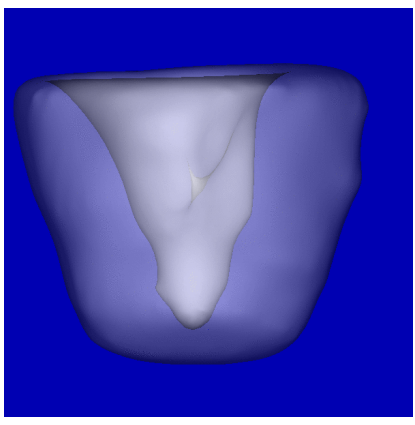

$(c)$

Fig. 1. (a), Objects of interest in LV images; (b), example of Left Ventricular Myocardium segmentation and $(c)$, a surface rendering.

than the one of $L V C$. The thickness of the $L V M$ is not known in advance. It can change from one patient to another and is neither constant around the chamber of a same patient. Nevertheless, the thickness of the $L V M$ can be bounded, and since we only consider live patients, it cannot be null. Contrary to the $E n B$, the $E p B$ is a smooth surface.

Last, the $L V B$ is composed of several tissues including among other the liver, the stomach, the right ventricle and the lungs. Some of them, such as the right ventricle or the lungs, have characteristic intensities and can be used as landmarks for the $L V B$.

The proposed segmentation method is composed of two consecutive steps which consist of $L V C$ segmentation, followed by $L V C M$ segmentation. Then, $L V M$ is simply the set difference between them. Since endo- and epicardial borders differ both in terms of contrast and shape, we segment them by different strategies.

\subsection{Endocardial border segmentation}

The segmentation of the $E n B$ is performed independently on each volume of the sequence. The idea is, first, to find a marker for the $L V C$ (i.e., a subset of the $L V C$ made of points which belong to the $L V C$ with certainty). Then, this marker is dilated conditionally to a second set made of points which possibly belong to the $L V C$.

The $L V C$ is made of a very light core object surrounded by a "ring" of lower intensity (see Fig. 1). The core object $C_{t}$ can be segmented, separately in each volume, by selecting the appropriate connected component of an upper-threshold $I_{t}\left[s_{1}\right]$ of the input image $I_{t}$ at level $s_{1}$, i.e., $I_{t}\left[s_{1}\right]=\left\{x \in V_{t} \mid I_{t}(x) \geq s_{1}\right\}$, where $V_{t}$ is the set of voxels of the t-th $3 \mathrm{D}$-image. In our experiments, the images are cropped such that, in each $3 \mathrm{D}$ volume, the center belongs to the core of $L V C$. To this aim, the user selects a single point roughly located at the center 
of the $L V C$ in one of the $3 \mathrm{D}$ images of the sequence. Then, in each $3 \mathrm{D}$ image, a box whose center is the specified point is automatically cropped. This step, which is the only user interaction of the whole method, could be automated but the physicians who made the evaluation prefer to keep this control point.

Thus, in the 3D graph (induced by the classical 6-adjacency relation) corresponding to the image under consideration, $C_{t}$ is the connected component of $I_{t}\left[s_{1}\right]$ which contains the center of the volume.

This core object is surrounded by a "ring" of lower intensity, still higher than the intensity of the $L V M$, which is a partial volume effect due the presence of both blood and muscle within the space of a single voxel. In order to get this ring segmented, we perform a second upper threshold $I_{t}\left[s_{2}\right]$ of $I_{t}$ at level $s_{2}<s_{1}$; $I_{t}\left[s_{2}\right]$ and $C_{t}$ are combined together thanks to a geodesic dilation of size $r_{1}$ of $C_{t}$ in $I_{t}\left[s_{2}\right]$ (see [10], chapter 6.1). In other words, $L V C_{t}$, the segmentation of the $L V C$ in the t-th volume of the $4 \mathrm{D}$ sequence, is the set of all points linked to $C_{t}$ by a path in $I_{t}\left[s_{2}\right]$ of length less than a predefined value $r_{1} \in \mathbb{N}$.

\subsection{Epicardial border segmentation}

In this section, we describe $L V C M$ segmentation. This segmentation is performed after the one of the $L V C$. It must preserve some anatomical constraints. Since we consider live patients, the left ventricular myocardium does not have any hole. This leads to the constraint $E p B \cap E n B=\emptyset$. In the graph framework assumed in this paper, it means that, in each $3 \mathrm{D}$ graph of the $4 \mathrm{D}$-sequence, the spatial neighborhood of $L V C_{t}$ should be included in $L V C M_{t}$, the segmentation of the $L V C M$ in the t-th image: $L V M$ must be at least one pixel thick.

The segmentation of $L V C M$ is performed thanks to a watershed-based procedure. Marker selection plays a primary role in the result of the watershed. In our application, it allows, in particular, to take into account the prior knowledge of the heart by imposing constraints on the markers. Since we want to get the separation between $L V C M$ and $L V B$, we need to select two markers, one for each of these objects. In the following, $m L V C M$ and $m L V B$ denote these two markers whereas $w L V C M$ and $w L V B$ denote the corresponding objects in the segmentation obtained through the watershed. The recognition process, described below, is performed independently on each volume of the $4 \mathrm{D}$-sequence. On the other hand, the watershed is applied on the $4 \mathrm{D}$-graph associated to the $4 \mathrm{D}$-sequence starting from the union of all the markers extracted in 3D.

$\boldsymbol{m} \boldsymbol{L} \boldsymbol{V} \boldsymbol{C} \boldsymbol{M}$ : Recognition of the $\boldsymbol{L} \boldsymbol{V} \boldsymbol{C} \boldsymbol{M}$ We first tackle the problem of computing $m L V C M_{t}$, for any given $t$. The watershed transform extends the markers as much as possible. Thus, by definition, each marker taken as an input of the watershed transform is included in the corresponding object obtained after the watershed. Hence, in order to fulfill the pre-cited inclusion constraint, we need to impose the inclusion of the spatial neighborhood of $L V C_{t}$ in $m L V C M_{t}$.

In order to compute this marker, the basic idea is to dilate the already known left ventricular chamber as much as possible while ensuring that the marker 
lies in the "true" $L V C M_{t}$. Since we are dealing with infarcted ventricles, it is not reasonable to use the same dilation parameter everywhere around the chamber and for every different dataset. Thus, the dilations must be constrained in order to fit each particular anatomy. To this aim, we introduce a definition of a constrained dilation, which, due to space restriction, is not provided in this paper but will be given in a forthcoming extended version.

In our application, the goal is to find a constraining set which is able to capture some of the features which are particular to the shape of the heart under consideration. Therefore, this set is extracted from the images. Some bright and dark regions (corresponding, for instance, to the right ventricle, the vascular network or the lungs) surrounds the $L V M$. These regions can be classified as non-myocardium objects and, thus, used as our constraining set. Some robust markers of these zones can be easily extracted by upper and lower threshold of the image: $\mathcal{T}_{t}=\left\{x \in V_{t} \backslash N_{t}\left(L V C_{t}\right) \mid I_{t}(x)<s_{3}\right.$ or $\left.I_{t}(x)>s_{4}\right\}$, where $N_{t}\left(L V C_{t}\right)$ denotes the dilation of size 1 of $L V C_{t}, s_{3} \in \mathbb{N}$ and $s_{4} \in \mathbb{N}$. Then, $m L V C M_{t}$, the marker of the $L V C M$ in the t-th $3 \mathrm{D}$ image is defined as the dilation of size $r_{2}$ of $N_{t}\left(L V C_{t}\right)$ constrained by $\mathcal{T}_{t}$. The constrained dilation is designed to take into account that we deal with markers of an object rather than with its precise segmentation. Thus, the set $\mathcal{T}_{t}$ does not need to be a precise segmentation; it is used as a landmark for the right ventricle, the vascular network, etc...

$\boldsymbol{m} \boldsymbol{L} \boldsymbol{V} \boldsymbol{B}$ : Recognition of the $\boldsymbol{L} \boldsymbol{V} \boldsymbol{B}$ We now explain how to compute, in each 3D image of the sequence, the marker for the left ventricular background $\left(m L V B_{t}\right)$. We consider the following facts: $(a)$, the thickness of a left ventricular myocardium cannot exceed a given threshold, denoted by $r_{3} ;(b)$ the set $\mathcal{T}_{t}$ is made of points which are in the background of the left ventricular chamber and myocardium. We deduce from fact $(a)$ that the points farther from $L V C_{t}$ than a distance of $r_{3}$ have to belong to $L V B_{t}$. This set is denoted by $L V B_{t}^{r_{3}}$. From fact $(b)$, we also want $\mathcal{T}_{t}$ to be included in $m L V B_{t}$. In order to satisfy these two requirements, the easiest way is to consider $m L V B_{t}$ as the union of $L V B_{t}^{r_{3}}$ and $\mathcal{T}_{t}$. We observe that this set is not necessarily connected. Using it as a watershed marker can induce some unwanted configurations of $w L V C M_{t}$, obtained after the watershed process. In particular, $w L V C M_{t}$ can have some cavities.

In order to overcome this problem, the idea is to derive $m L V B_{t}$ by a topologypreserving transformation. It is straightforward that the left ventricular background contains a unique cavity made of the left ventricular chamber and myocardium. We remark that $m L V C M_{t}$ is, by construction, connected and that it must be surrounded by $L V B_{t}$. The complementary set of $m L V C M_{t}$ thus contains a unique cavity, and obviously, surrounds $m L V C M_{t}$. Therefore, we choose to derive $m L V B_{t}$ from the complementary set of $m L V C M_{t}$. The idea is to reduce this set while both preserving its topology and respecting facts $(a)$ and $(b)$. To this aim, we use a constrained ultimate homotopic skeleton [12]. Roughly speaking, an ultimate homotopic skeleton of a set $X$ constrained by a set $C$, has the same topology as $X$, contains $C$, and cannot be reduced (by point removal) 
while keeping these two invariants. Thus, we compute $m L V B_{t}$, the marker of the left ventricular background, as the skeleton of $\overline{m L V C M_{t}}$ (i.e., the complementary set of $m L V C M_{t}$ ) constrained by $\mathcal{T}_{t} \cup L V B_{t}^{r_{3}}$. We finally obtain a set $m L V B_{t}$ which has the desired topology and which respects facts (a) and (b).

Delineation The watershed transformation [11] is used as a fundamental step in many powerful segmentation procedures. It is a graph-based method that allows, from a gradient image, to find a "thin" separation between the components of a given set of points called marker. In this application, we use the watershed approach [13] that we have developed since it has good robustness properties [14]. We consider the $4 \mathrm{D}$ graph corresponding to the $3 \mathrm{D}+\mathrm{t}$ sequence. The neighborhood of each voxel corresponds to its 6 neighbors in 3D and the voxels just before and after in the time sequence. In this graph, the watershed of the $4 \mathrm{D}$ gradient magnitude image is computed, starting from the two markers made by the union of all $m L V C M_{t}$ and $m L V B_{t}$. As a result, we obtain, a first segmentation of $L V C M$, denoted $w L V C M$ lying on a background object $w L V B$.

Smoothing In order to obtain the final segmentation of $L V C M$, we use shape filters coming from mathematical morphology. An alternating sequential filter $(A S F)$ with parameters $r$ and $r^{\prime}$ is a sequence of intermixed openings and closings by balls of increasing size, where $r$ and $r^{\prime}$ specify the size of respectively the smallest and largest ball (see [10], chapter 8.3). An opening of a set $X$ by a ball of radius $r$ is the union of all balls of radius $r$ which are included in $X$. On the other hand, the closing of $X$ is the complementary of the opening of $\bar{X}$. Thus, an $A S F$ smoothes the object and its complementary in a balanced way while preserving the "most significant balls" of both object and background.

The endocardial border can be modeled as the surface of a cone. Each section (perpendicular to the height axis) of a cone is a disc. Then, we consider 2dimensional $A S F$, with parameters $r_{4}$ and $r_{5}$, for smoothing each 2D section of the $3 \mathrm{D} w L V C M_{t}$.

\section{Experiments}

\subsection{Image acquisition}

In our experiment, the original images consist of short axis $2 \mathrm{D}$ sequences (between 8 and 13 sequences of 25 images per patient) with breath-held, ECG-gated acquisitions from base to apex. The most basal slices included in the analysis was located lust above mitral valve within LVC. To be included, the basal myocardium had to be visible in the entire circumference at end-systole. The most apical slice was chosen as the one with the smallest visible LVC at end-systole. The sequences are registered on the heart-cycle, and thus they can be stacked to construct 3D sequences. Each 3D sequence is made of 25 volumes and covers a complete heart cycle. The spatial resolution is typically $1,7 \times 1,7 \times 6 \mathrm{~mm}^{3}$. 
Before applying the segmentation procedure, the images are over-sampled in order to get isotropic voxels. When a misalignment of the different sections of a same volume is observed, a basic registration procedure is possibly applied as a preprocessing step.

\subsection{Parameters}

In our method, there are two kinds of parameters. A first series is related with the shape of a left ventricle and can be expressed by distance measures. In our experiment, $r_{1}, r_{2}, r_{3}, r_{4}$ and $r_{5}$ were set up to respectively $9,5,25,7$ and $12 \mathrm{~mm}$. These parameters are neither patient-specific nor device-specific. On the other hand, our method comprises a series of parameters relative to the intensity of the images. In our experiment, $s_{1}, s_{2}, s_{3}, s_{4}$ were set up to respectively 170,105 , 20,170 . These parameters are not patient-specific but they are device-dependent which means that they must be re-estimated for each device.

The proposed method relies on a succession of operators. The results of each of these operators are well specified with respect to anatomical constraint. Thus, each of them can be tuned separately: the operators -and, thus, the parametersare independent. One of the two cardiologists estimates these parameters by independently tuning each operator thanks to qualitative assessment. This choice is the less time-consuming one since it does not require manual segmentation and minimizes the number of tests to be performed. For instance, $s_{1}$ is estimated by interactively thresholding the image: the highest threshold such that all points (above this threshold) connected to the center of the 3D volume belong to the $L V C$ with certainty is kept. We emphasize that only 3 training datasets were enough to set up these parameters. Their complete tuning took four hours of work for the practician. We recall that if one wish to use the proposed method on a different device, only the parameters relative to image intensity need to be re-calibrated. This would require less than one hour.

Furthermore, during the setting of the parameters, we noticed their robustness with respect to small variations of typically, $\pm 5 \%$ for the parameters relative to the intensity of the images and $\pm 10 \%$ for the shape parameters. In a forthcoming extended version, we intend to make a systematic study of the impact of these small variations on the resulting segmentations.

\subsection{Results}

Quantitative assessment Four-dimensional cardiac cine-MR images were acquired in 18 consecutive patients which were not specifically chosen for this application. Images were processed automatically by the proposed 4D method. In Fig. 1, we show (a) the internal border (on 3 orthogonal sections of a 3D volume) of the segmented $L V M$, obtained by the $4 \mathrm{D}$-method, superimposed to the corresponding sections of the original image and $(b)$ a surface rendering of the segmentation. For comparison, the dataset was also manually segmented by 2 independent experts (cardiologists), called $e_{1}$ and $e_{2}$ in the sequel. On each 2D 
section of the 3D volumes corresponding to end-diastolic and end-systolic time, they manually delineated $E n B$ and $E p B$.

Given two surfaces $\partial X$ and $\partial Y$ represented by two sets of polygons, the pointto-surface measurement (P2S) between $\partial X$ and $\partial Y$ estimates the mean distance between the vertices of $\partial X$ and $\partial Y$. A symmetrical measure is obtained by taking the maximum from the P2S between $\partial X$ and $\partial Y$ and the P2S between $\partial Y$ and $\partial X$.

The P2S achieved by other groups for segmenting the endocardial and epicardial border on their datasets are presented in Table 1a.

On our dataset, the surfaces $(E n B$ and $E p B)$ are extracted from the segmentations by a marching cube algorithm. The P2S was performed between the segmentations obtained by the software and by the two experts. In order to evaluate the inter-observer variability the P2S between the two experts is also provided. Table $1 \mathrm{~b}$ present the mean and standard deviation of these measures at end-diastolic time and end-systolic time. We note that, in all cases of Table 1b, the P2S is less than 1 pixel. The method achieved a mean P2S of $1.51 \mathrm{~mm} \pm 0.38$ for the endocardial border and a mean P2S of $1.81 \mathrm{~mm} \pm 0.43$ for the epicardial border. These results compare favorably with the results obtained by other groups on their own datasets (see Table 1b). Furthermore, the P2S between automatic and manual segmentations is in the same range as the inter-observer P2S: the proposed software produces satisfying segmentations.

Table 1. (a), Point to surface measurements from the results of different cardiac segmentation methods. (b), More details on the segmentations obtained by our method, (mean point to surface in $\mathrm{mm} \pm$ standard deviation).

\begin{tabular}{|c|c|c|}
\hline \multicolumn{3}{|c|}{ (a) } \\
\hline & $E n B$ & $E p B$ \\
\hline [6] & $2.01 \pm 0.31$ & $2.77 \pm 0.49$ \\
\hline [4] & $2.75 \pm 0.86$ & $2.63 \pm 0.76$ \\
\hline [5] & $2.28 \pm 0.93$ & $2.62 \pm 0.75$ \\
\hline [8] & $1.88 \pm 2.00$ & $2.75 \pm 2.62$ \\
\hline$[7]$ & $1.97 \pm 0.54$ & $2.23 \pm 0.46$ \\
\hline ours & $1.51 \pm 0.38$ & $1.81 \pm 0.43$ \\
\hline
\end{tabular}

\begin{tabular}{|l|l|l|c|}
\hline & $(b)$ \\
\hline End-diastolic time & soft. vs. $e_{1}$ & soft. vs. $e_{2}$ & $e_{1}$ vs. $e_{2}$ \\
$E n B$ & $1.52 \pm 0.35$ & $1.67 \pm 0.43$ & $1.37 \pm 0.47$ \\
$E p B$ & $2.04 \pm 0.35$ & $1.68 \pm 0.39$ & $1.23 \pm 0.41$ \\
End-systolic time & & & \\
$E n B$ & $1.50 \pm 0.41$ & $1.35 \pm 0.34$ & $1.15 \pm 0.41$ \\
$E p B$ & $1.90 \pm 0.56$ & $1.61 \pm 0.41$ & $1.31 \pm 0.83$ \\
\hline
\end{tabular}

Left ventricular ejection fraction (EF) and left ventricular myocardium mass (MM) are critical parameters for cardiac diagnosis and remodeling prevention. Their estimation is routinely used by cardiologists. The EF is the amount of blood ejected during a heart cycle expressed as a fraction of the tele-diastolic volume. In our dataset the EF (resp. MM) range was 20-75\% (resp. 94-197 g). From the segmented images, the EF can be simply computed by $\left(\left|L V C_{\max }\right|-\right.$ $\left.\left|L V C_{\min }\right|\right) /\left|L V C_{\max }\right|$, where $\left|L V C_{\max }\right|$ (resp. $\left|L V C_{\min }\right|$ ) is the maximal (resp. minimal) volume of the left ventricular chamber along the heart cycle. Let $X_{p}^{o}$ denote the measure of the parameter $X$ performed by operator $o$ for patient $p$, where $X \in\{E F, M M\}, o \in\left\{e_{1}, e_{2}, s\right\}$, and $p \in[1,18]$. We take ref $X_{p}=$ $\left(X_{p}^{e_{1}}+X_{p}^{e_{2}}\right) / 2$ as a reference value for the parameter $X$ on patient $p$ and we evaluate the deviation $\Delta X_{p}^{o}=\left|X_{p}^{o}-\operatorname{ref} X_{p}\right| / \operatorname{ref} X_{p}$. Notice that $\Delta X_{p}^{e_{1}}=\Delta X_{p}^{e_{2}}$. On 18 patients, our automatic software achieved a mean deviation on the EF (resp. MM) of 0.032 (resp. 0.050) whereas the experts achieved 0.055 (resp. 
0.052). Thus, the proposed tool produces reliable assessment of left ventricular functional parameters.

In an extended version of this paper, we provide further experimental results, including, in particular, a qualitative assessment of the method.

3D watershed vs. 4D watershed The proposed segmentation scheme has been tested using the 4D watershed-based procedure described above and also with a variant using only $3 \mathrm{D}$ watersheds (one per volume $V_{t}$ ). It is visible on the computer screen that, contrary to the $3 \mathrm{D}$ variant, the $4 \mathrm{D}$ algorithm helps keeping the temporal coherency between successive segmentations along the heart cycle. In order to precisely evaluate this temporal coherency, we compute, for both methods, the P2S between successive $E p B$ s along the cardiac cycle. The means of these measurements among all patients are plotted along the cardiac cycle in Fig. 2. We observe that both curves have the same shape but that there is a nearly constant difference between them. Thus, this confirms the fact that segmentations obtained by the $4 \mathrm{D}$ method are more regular.

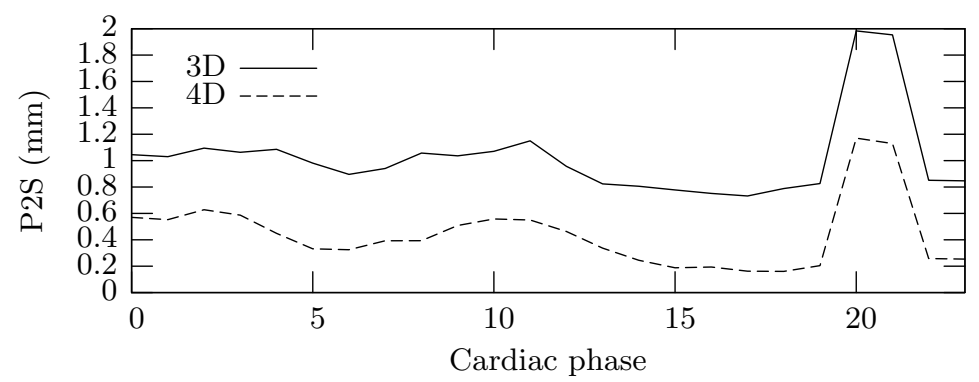

Fig. 2. The point to surface measurements between the successive $E p B$ s along the cardiac cycle.

The segmentation of endocardial borders is performed independently on each $3 \mathrm{D}$ volume of the sequence. Nevertheless, we have not noticed any regularity problem in the EnBs produced by the automated software. This explains why we have not developed a $4 \mathrm{D}$ procedure to segment the $L V C$.

\section{Conclusion and perspectives}

In this paper, we propose a fast (worst computational time among the 18 datasets is 4'3" on a low-end computer laptop) and automated procedure to segment the left ventricular myocardium in $4 \mathrm{D}$ cine-MRI sequences, taking into account spatiotemporal properties. This procedure can be used in clinical routine. Thanks to the comparison with manual segmentations performed by two cardiologists, 
we demonstrated the accuracy of the proposed method and the relevance of the ejection fraction and myocardium mass derived from the automated segmentations.

\section{References}

1. Buser, P.T., Auffermann, W., Holt, W.W., Wagner, S., Kircher, B., Wolfe, C., Higgins, C.B.: Noninvasive evaluation of global left ventricular function with use of cine nuclear magnetic resonance. Journal of the American College of Cardiology 2(13) (1989) 1294-1300

2. McInerney, T., Terzopoulos, D.: A dynamic finite element surface model for segmentation and tracking in multidimensional medical images with application to cardiac 4D image analysis. Computerized Medical Imaging and Graphics 19(1) (1995) 69-83

3. van der Geest, R.J., Buller, V.G.M., Jansen, E., Lamb, H.J., Baur, L.H.B., van der Wall, E.E., de Roos, A., Reiber, J.H.C.: Comparison between manual and semiautomated analysis of left ventricular parameters from short-axis MR images. Journal of Computer Assisted Tomography 21 (1997) 756-765

4. Mitchell, S.C., Lelieveldt, B.P.F., van der Geest, R.J., Bosch, H.G., Reiber, J.H.C., Sonka, M.: Multistage hybrid active appearance model matching: Segmentation of left and right ventricles in cardiac $\mathrm{mr}$ images. IEEE Trans. on Medical Imaging 20(5) (2001) 415-423

5. Kaus, M.R., von Berg, J., Weese, J., Niessen, W., Pekar, V.: Automated segmentation of the left ventricle in cardiac MRI. Medical Image Analysis 8 (2004) $245-254$

6. Lötjönen, J., Kivistö, S., Koikkalainen, J., Smutek, D., Lauerma, K.: Statistical shape model of atria, ventricles and epicardium from short- and long-axis MR images. Medical Image Analysis 8 (2004) 371-386

7. van Assen, H., Danilouchkine, M.G., Frangi, A.F., Ordàs, S., Westenberg, J.J.M., Reiber, J.H.C., Lelieveldt, B.P.F.: SPASM: A 3D-ASM for segmentation of sparse and arbitrarily oriented cardiac MRI data. Medical Image Analysis 10 (2006) 286-303

8. Lorenzo-Valdés, M., Sanchez-Ortiz, G.I., Elington, A.G., Mohiaddin, R.H., Rueckert, D.: Segmentation of 4D cardiac MR images using a probabilistic atlas and the EM algorithm. Medical Image Analysis 10 (2006) 286-303

9. Montagnat, J., Delingette, H.: 4D deformable models with temporal constraints: application to $4 \mathrm{D}$ cardiac image segmentation. Medical Image Analysis 9 (2005) 87-100

10. Soille, P.: Morphological Image Analysis. Springer-Verlag (1999)

11. Beucher, S., Meyer, F.: The morphological approach to segmentation: the watershed transformation. E. Dougherty (Ed.), Mathematical Morphology in Image Processing, Marcel Decker (1993) 443-481

12. Couprie, M., Coeurjolly, D., Zrour, R.: Discrete bisector function and Euclidean skeleton in 2D and 3D. Image and Vision Computing (2006) to appear.

13. Couprie, M., Najman, L., Bertrand, G.: Quasi-linear algorithms for the topological watershed. Journal of Mathematical Imaging and Vision 22(2-3) (2005) 231-249

14. Najman, L., Couprie, M., Bertrand, G.: Watersheds, mosaics and the emergence paradigm. Discrete Applied Mathematics 147(2-3) (2005) 301-324 